Research Article

\title{
Experimental Study on Interaction between Nanosecond Pulsed Laser and Normal Shock Wave
}

\author{
Jilin Shi $\mathbb{D}^{D}$, Diankai Wang $\mathbb{D}^{D}$, and Longcheng Huang \\ Space Engineering University, State Key Laboratory of Laser Propulsion and Application, Beijing 101416, China \\ Correspondence should be addressed to Diankai Wang; 274233405@qq.com
}

Received 7 May 2021; Revised 8 July 2021; Accepted 14 August 2021; Published 20 August 2021

Academic Editor: Chao Tao

Copyright ( 92021 Jilin Shi et al. This is an open access article distributed under the Creative Commons Attribution License, which permits unrestricted use, distribution, and reproduction in any medium, provided the original work is properly cited.

\begin{abstract}
Nanosecond pulsed lasers possess two remarkable advantages: a high peak power density and the ability to break down air to form plasma readily. Therefore, they have significant practical value in the drag reduction of a supersonic body. An experimental investigation is conducted on the fundamental physical phenomenon of the interaction of the pulsed laser plasma with a normal shock wave to reveal the mechanism of drag reduction. Moreover, a high-precision schlieren system is developed to measure complex wave structures with a time resolution of up to $30 \mathrm{~ns}$ and a spatial resolution up to $1 \mathrm{~mm}$. A high-speed particle image velocimetry system is set up to measure the velocity and vorticity of the flow field quantitatively; the system has a time resolution of up to $500 \mathrm{~ns}$. The characteristics of the spherical shock wave and the high-temperature and low-density region induced by the laser plasma are presented. The flow characteristics and evolution process of the laser plasma under a normal shock wave are substantially revealed. The cause of the supersonic drag reduction by the pulsed laser plasma is illustrated with numerical simulation results. The following results are obtained in this study: the initial Mach number of the shock wave induced by the laser plasma increases with the laser energy, and the shape of the wave gradually evolves from a droplet shape to a spherical shape. The propagation velocity decreases with time and is close to the sound velocity after $50 \mu \mathrm{s}$. The shape of the initial high-temperature and low-density region is approximately spherical; it subsequently destabilizes to form a sharp spike structure in the laser's incident direction. Ultimately, the region evolves into a double-vortex ring structure with upper and lower symmetry; the size of this region increases with the laser energy.
\end{abstract}

\section{Introduction}

Among the active flow control technologies that have been applied to the supersonic body, pulsed laser plasma has become worthy of special attention because of its good directivity, fast activation speed, strong controllability, and high peak power density. It has broad practical prospects in terms of drag reduction, boundary layer transition, and intake capture.

In 1978, Myrabo [1] first proposed the concept of a Directed-Energy Air-Spike. High-frequency laser energy deposition could produce a series of plasma hot cores, and the interaction between hot cores and bow shock formed an air spike to reduce the drag on the surface of the aircraft. The schematic illustration of the energy deposit technique for supersonic drag reduction is shown in Figure 1. On the center axis of the blunt body, the hot cores and shock waves generated by pulse energy deposition flow downstream with the supersonic flow. When the hot cores interact with the bow shock, the bow shock will be reconstructed, and the stagnation point pressure will decrease. It is similar to the drag reduction method used for the front end of the supersonic body and the reverse jet [2, 3]. In 1994, Tret'yakov et al. $[4,5]$ first realized the high-repetition laser energy deposition in a supersonic argon gas flow. They reported that the mechanism of wave drag reduction is the formation of a hot wake after the laser energy deposition and the decrease of the gas density upstream of a supersonic body. Adelgren et al. $[6,7]$ controlled the flow field by the upstream singlepulse laser energy deposition in quiescent air, the bow shock with Mach of 3.45, and the type IV shock interaction. The experimental results showed that the peak pressure of the 


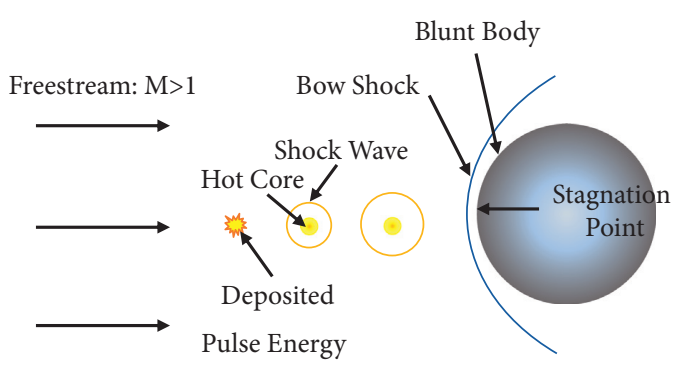

(a)

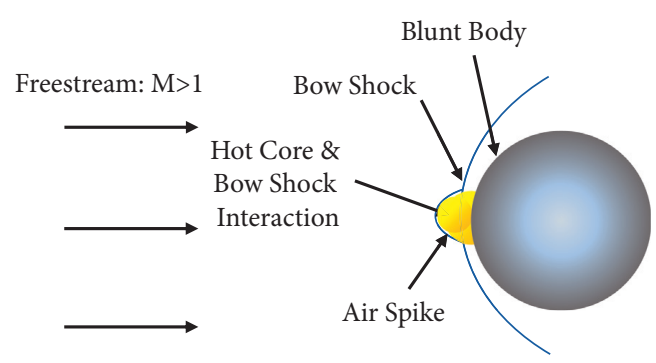

(b)

FIgURE 1: Schematic of interaction between high-frequency laser energy deposition and bow shock. (a) Initial deposition. (b) Hot core and bow shock interaction.

bow shock and the type IV shock interference was reduced by $40 \%$ and $30 \%$, respectively. Minucci et al. [8-11] conducted a single-pulse laser drag reduction experiment in a hypersonic shock wave tunnel. The air spike structure was observed using direct photography. The schlieren photography showed the plasma generation and convection and the perturbation of the flow field from the shock wave induced by laser energy deposition. Riggins et al. [12] reported that the mechanism of energy deposition to drag reduction was that the laser energy adjusts and weakens the bow shock before the blunt body, which changed the flow field structure and reduced the wave drag. Taguchi et al. [13] reported that the reason for the decrease of the wave drag is the reduction of the surface pressure of the blunt body. Ogino et al. [14] reported that the baroclinic vortex induced by Richtmyer-Meshkov instability caused a low entropy shock wave in the flow field, thereby resulting in wave drag reduction. Yu and Yan $[15,16]$ studied the effect of single-pulse laser energy on a blunt body's surface pressure in the Mach 8 flow field and explained the pressure variation curve of the stagnation point in the front of the blunt body. They proposed that the pressure decrease was due to the low-pressure zone following the shock wave. Since 2010, Hong et al.'s $[17,18]$ research group has adopted a single-pulse and highrepetition-frequency nanosecond laser energy injection method to form a relatively stable quasi-static wave structure with lower total laser energy. A continuous low-density channel was formed on the surface of the blunt body. The repetitive laser energy injection deformed the bow shock wave into a structure similar to the oblique shock wave structure, which could significantly reduce the wave drag of the blunt body. However, the cause of the low-density channel formation was not explained. Sasoh et al. $[19,20]$ reported that when the hot core interacted with the bow shock wave, the density gradient of the hot core area was inconsistent with the pressure gradient direction, which meant there is a baroclinic gradient [21]. Moreover, the baroclinic gradient led to the deposition of vorticity in this area and would accelerate the generation of turbulence. The wave drag reduced because the local bow shock weakened.

One of the fundamental physical phenomena of the pulsed laser plasma interacting with the supersonic flow field is the laser-plasma interaction with the shock wave. However, the interaction between the laser plasma and a bow shock is a complex physical problem, which involves laser energy transmission and deposition, plasma formation by ionized air, energy and mass transfer in air, and the evolution of plasma under shock. Thus, it is a complex highspeed unsteady process. In addition, the quantitative measurements of the flow field details are currently insufficient. The experimental results are mainly based on macroscopic observations of the bow shock deformation and changes in the surface pressure and resistance of the blunt body. These observations limit a deeper understanding of the laser drag reduction mechanism.

The interaction between the laser and the bow shock starts from the most upstream front end portion of the bow shock, which is critical to reveal the drag reduction mechanism. However, due to the short distance between the bow shock and the blunt body, the transmitted shock wave generated during the interaction is quickly reflected, causing significant interference to the flow field. This adds to the difficulty of identifying the drag reduction mechanism. On the contrary, the characteristics of the front end portion of the bow shock are similar to the steady normal shock wave from aerodynamics. This paper intends to simplify the bow shock to the steady normal shock as the research object as a first step in exploring this complicated issue. In this paper, the research focuses on the nanosecond pulsed laser ionized air in the experimental section of the shock tube. The particle image velocimetry (PIV) diagnostic technique was used to study the flow field velocity as well as the mechanism of the vorticity evolution process; the high-precision schlieren system was used to study the evolution of the flow field structure of the interaction between the nanosecond laser and the normal shock wave.

The initial step of the experimental research was carried out in quiescent air to understand the evolution of the spherical shock wave and the ion field. The ion field is in the ionized region after the laser energy is focused on the ionized air. Subsequently, the mechanism and the law of interaction between the nanosecond pulsed laser and the quiescent air were identified and summarized. Experiments were conducted in the shock tube to investigate the detailed evolution mechanism of the shock structure during the interaction process. The results of these experiments reveal the flow characteristics during the interaction between the laser plasma and the normal shock wave. Therefore, they provide 
significant evidence for revealing the mechanism of pulsed laser drag reduction.

\section{Key Experimental Techniques}

2.1. Shock Tube Experimental Technique. The experiments were conducted in a shock tube with a rectangular cross section. The high-pressure gas was used to break the diaphragm and generate a steady incident normal shock wave. The desired incident shock wave velocity was realized with two settings: the initial gas component of the high-pressure section of the shock tube and the pressure ratio of the highand low-pressure sections. The high-pressure section was driven by nitrogen. The rupture pressure of the plastic diaphragm was about $0.9 \mathrm{MPa}$ to generate a normal shock wave with a Mach number of 1.45 . The rupture pressure of the aluminum diaphragm was about $2 \mathrm{MPa}$ to generate a normal shock wave with a Mach number of 1.75 . The nanosecond pulsed laser energy was injected when the normal shock wave reached the experimental position, and the ionized air formed a plasma. The normal shock wave and the plasma interacted at the experimental position, and typical photographs of the interaction process were obtained using the high-precision schlieren system and the PIV system. Figure 2 shows the experimental design.

The shock tube, lasers, and measurement systems are all pulsed modes, and timing synchronization is a crucial experimental technique. Two PCB sensors were installed in the low-pressure section of the shock tube to monitor the incident shock speed and output a timing trigger signal to the DG645 Digital Delay Generator, which is a precision 8channel delay generator that can output arbitrary delays from 0 to $2000 \mathrm{~s}$ with five ps resolution and typical RMS jitter of 12 ps. DG645 is the core component to realize the timing synchronization of the subsystems. It outputted the trigger signal to the nanosecond pulse laser, the flash source of the schlieren system, the PIV system laser, and the high-speed camera. Control signals from the DG645 ensure that when the normal shock wave reaches the experimental observation window, the nanosecond pulse laser has focused on the ionized air to form a plasma and simultaneously turns on the flash of the measurement system and the high-speed camera to record the flow field evolution process. Figure 3 illustrates the timing synchronization control scheme. It should be noted that the experiments of the schlieren system and the PIV system were conducted separately.

2.2. High-Precision Schlieren Experimental Technique. The schlieren is a traditional measurement method, which adopts the "Z" optical path layout. With the ongoing development of technologies for light sources and high-speed photography, schlieren measurement still has much room for improvement in resolution and sensitivity. To improve the spatial and temporal resolution as well as the system sensitivity, the following three technical approaches are proposed and implemented:

(1) A high-brightness pulsed xenon flash source was selected. The illumination phenomenon with a high

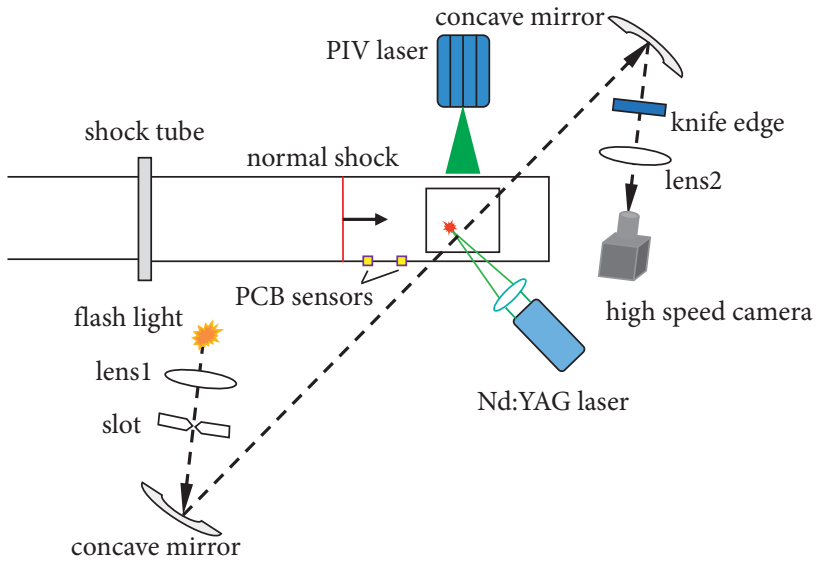

Figure 2: Experimental design of the shock tube.

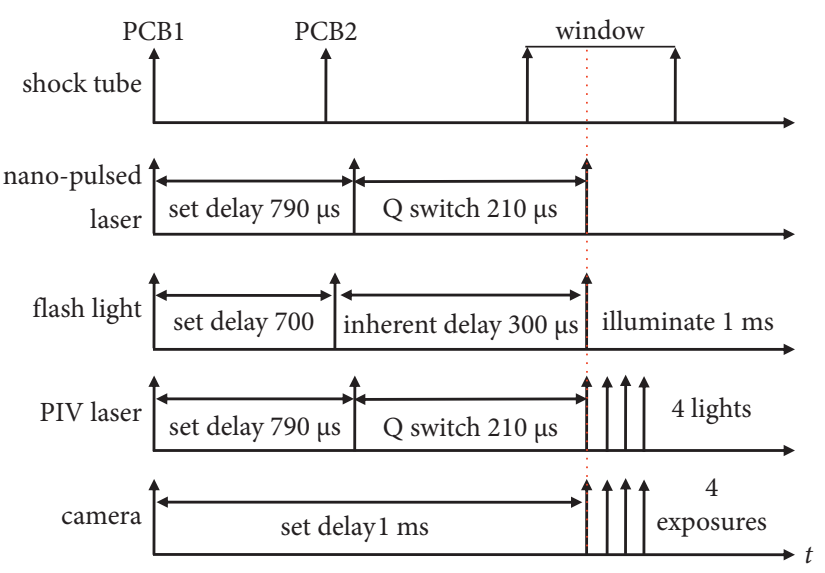

Figure 3: Timing synchronization control scheme.

voltage breakdown of xenon gas is characterized by high brightness. A bright field of view can be obtained by exposure of $30 \mathrm{~ns}$ during the duration of the light source emission, which meets the requirements of short exposure and high time resolution. As long as the synchronization problem is solved for fast processes, the photography requirements can be met.

(2) The light source was shaped. Compared with the shock wave, the weak flows (e.g., vortex structure and sparse wave) impose extremely high requirements on the measurement system's sensitivity. Thus, the light source must be shaped. The core component of the shaping optical path is a convex lens group. This group ensures that the light-emitting area has the highest brightness of the light source directed on the slit and takes the slit position as the system light source position. Moreover, the slit width is adjustable. The advantage of this method is that the light source is limited to a narrow space of a $0.5 \mathrm{~mm} \times 20 \mathrm{~mm}$ rectangle. A clear response can be obtained with a slightly light deflection, which can significantly improve the system sensitivity. 
(3) Solved the problem of synchronization and highspeed photography. The knife-edge was located at the focus of the lenticular mirror, and it matches the light source. The convex lens formed a clear image on the high-speed camera's receiving screen. In this paper, an HSFC PRO ultrahigh-speed enhanced camera by the German PCO company was employed. The minimum exposure time is up to $3 \mathrm{~ns}$ with a resolution of $1280 \times 1024$ pixels. Four channels share the trigger; they can work independently, where one photo per channel is recorded in each exposure. This enhanced camera effectively solves the problem in which the frame rate of a high-speed camera is difficult to increase, and the exposure time is challenging to shorten: high speed and high resolution are both realized. The color schlieren technology was also used in this paper to display the rich flow of details and continuous recordings. The homemade color filters are employed instead of traditional schlieren edges. Moreover, a V711 highspeed color camera by Vision Research Company was used as well. The shortest exposure time is $0.28 \mu \mathrm{s}$, and the camera frame rate is $40,000 \mathrm{fps}$ at a resolution of $800 \times 208$ pixels, which can be continuously used to record for approximately 1 second.

2.3. Fast PIV Experimental Technique. To quantitatively measure the velocity field, a fast PIV measurement technique was adopted in this paper. A trade-off exists between the pulse energy and the repetitive frequency; they are challenging to increase simultaneously. Therefore, a combination method was applied in this project to combine four independent Nd: YAG lasers. It controls the flow of four lasers according to timing requirements, ensuring the highest possible single pulse energy to illuminate the flow field and increase the repetition rate. The four-cavity laser used in this paper has single-pulse energy of up to $400 \mathrm{~mJ}$. The shortest time interval is $500 \mathrm{~ns}$, with an exposure time of $8 \mathrm{~ns}$ and a $532 \mathrm{~nm}$ laser wavelength. The lasershaping optical path shapes the excited cylindrical laser beam into a piece of light, illuminates the nano-trace particles in the experimental section of the shock tube, and records particles' scattering time-series photos using the HSFC PRO ultrahighspeed enhanced camera. $\mathrm{TiO}_{2}$ powder with a particle diameter of $50 \mathrm{~nm}$ was adopted as the trace particles. Figure 4 shows the nanoparticle generator. A copper filter with a pore size of $0.2-0.3 \mathrm{~mm}$ was installed at $c$ and $d$, and the $\mathrm{TiO}_{2}$ particle powder was placed on filter $c$ before the experiment. The highpressure gas was injected from the bottom channel $a$ and the middle channel $b$ to disperse the $\mathrm{TiO}_{2}$ particles and lift them upwards. Through filter $d$, the $\mathrm{TiO}_{2}$ particles were filtered again. $\mathrm{TiO}_{2}$ particles were ejected from the top of the particle generator into the experimental section.

\section{Experimental Results and Analysis}

3.1. Characteristics of the Pulsed Laser Plasma. The interaction between the laser and the flow field consists of two essential elements: the shock wave (generated by ionizing the

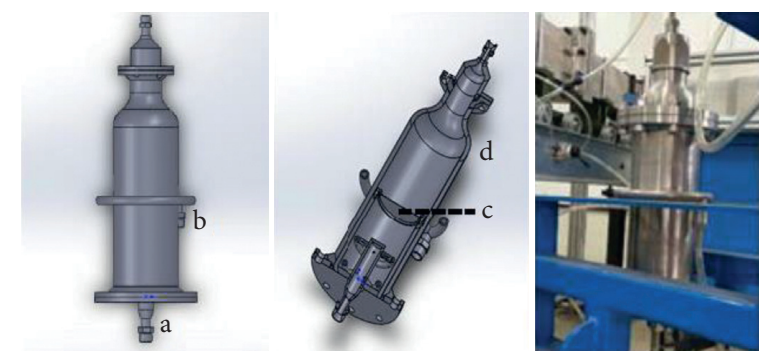

FIGURE 4: Design drawings and physical photos of the nanoparticle generator.

air) and the region of high temperature and low density. It should be noted that the laser pulse width is on the order of nanoseconds. The time scale of the single-pulsed laser energy deposition and plasma lifetime is on the order of nanoseconds. The time scale of the interaction between the laser and the flow field is on the order of microseconds. Therefore, to reveal the detailed flow mechanism, the main study in this paper is placed on the flow field changing process, which occurs after the laser-plasma generation. We decided to compare the evolution of a laser-produced plasma with and without a shock wave. The single-pulse nanosecond $\mathrm{Nd}$ : YAG laser is firstly deposited in quiescent air, and the high temporal-spatial resolution schlieren technique is also applied. The experimental study was conducted on developing the shock wave propagation velocity and the shape-changing of the high-temperature and low-density regions.

3.1.1. Characteristics of Shock Waves Induced by the Pulsed Laser Plasma. The schlieren photos of the flow field after the ionization of air by single-pulse laser energy are shown in Figure 5. The laser energy is $209 \mathrm{~mJ}$, the pulse width is eight $\mathrm{ns}$, the laser is incident from left to right, the air is quiescent with normal temperature and pressure, and the resolution is 22 pixels $/ \mathrm{mm}$ schlieren photo. The short black line in the images represents a length of $10 \mathrm{~mm}$. At $t=100 \mathrm{~ns}$, the white region of the ionization position is plasma. Due to the shielding phenomenon of the laser plasma, it can be seen that the region where the laser energy ionizes the air is not an ideal point, spherical shape, or an ellipsoid. The symmetrical shape is similar to water droplets. The deposited laser energy is higher on the left side, so the plasma region is larger, and the tip is formed on the right side. At this time, the laserinduced shock has not been separated from the plasma. At $t=400 \mathrm{~ns}$, the plasma has been quenched, and the shock front has been formed. It can be seen that the initial shape of the shock wavefront caused by the laser plasma is also a droplet shape. Between $t=1 \mu \mathrm{s}$ and $t=15 \mu \mathrm{s}$, the shock wave continuously propagates outward from the initial droplet shape to form the spherical shape; an air bubble similar to a spherical shape is formed at the ionization position. Due to the high-temperature characteristics of the laser plasma, it is assumed that the air bubble is heated by the laser plasma and has the characteristics of high temperature and low density. After $t=20 \mu$ s, the shock front is substantially spherical, and the high-temperature low-density air bubble shape remains approximately spherical. 

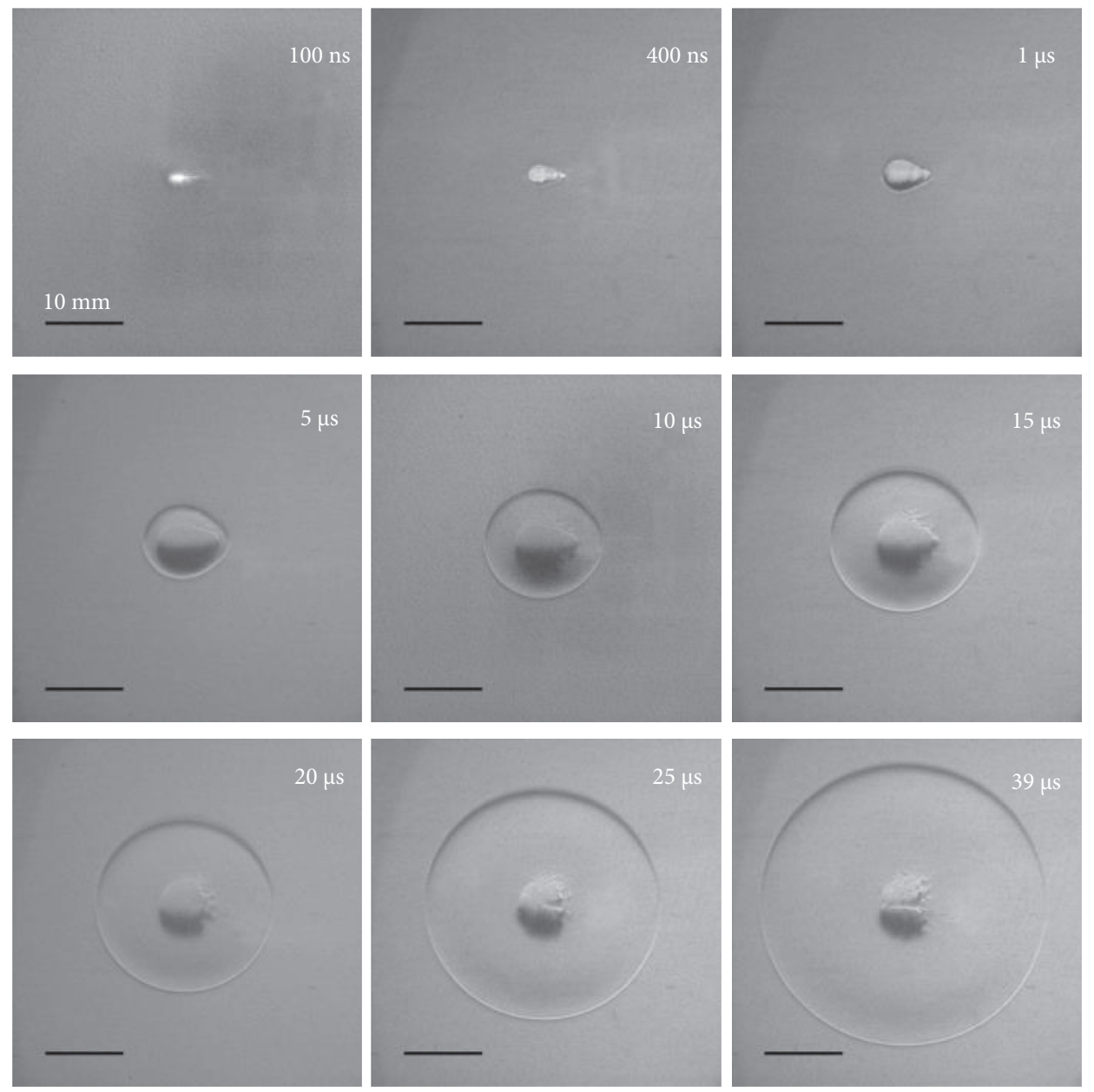

FIGURE 5: Schlieren photographs of quiescent air ionization by a single-pulse laser.

Figure 6 shows a comparison of the shock propagation's Mach number under different incident laser energies. For convenience, the longitudinal propagation Mach number $M_{y}$ is selected for comparison. The larger the incident laser energy, the larger the initial Mach number of the shock wave. When the single pulse energy reaches $152 \mathrm{~mJ}$, the initial Mach number reaches 4.1; when the single pulse energy reaches $56 \mathrm{~mJ}$, the initial Mach number reaches 3.4. The development of the shock waves, caused by different incident laser energies, is similar. The propagation velocity decreases with time, approaching the speed of sound after $t=50 \mu \mathrm{s}$.

\subsubsection{Characteristics of a High-Temperature and Low-Den-} sity Region. Figure 7 shows the results of the schlieren experiment in the high-temperature and low-density regions. At $t=18 \mu \mathrm{s}$, the high-temperature and low-density region is an approximately spherical shape. It is worth noting that the interface on the left side is relatively smooth. The instability first appears as wrinkles on the right side, which is downstream of the laser direction. The reason is that the laser plasma has a shielding effect, resulting in nonuniform energy deposition. Less laser energy is deposited at the right side, so the state change of the flow field is relatively small. The flow is less steady and is more susceptible to the outside higher density air, which penetrates the high-temperature and low-density region to form a spike.

Meanwhile, the air in the high-temperature and lowdensity region enters the outside air to form a bubble. The spike and the bubble collectively appear as wrinkles. With the superposition of disturbances, the instability is more severe. At $t=22 \mu \mathrm{s}$, the right side begins to collapse, and the outside air penetrates the high-temperature low-density region from right to left. After that, the outside air gradually penetrates the left portion of the high-temperature lowdensity region. At $t=60 \mu \mathrm{s}$, it completely penetrates to form a "plasma spike" and continues to flow upstream. At $t=150 \mu \mathrm{s}$, the high-temperature low-density region has evolved into a double-vortex ring structure with upper and lower symmetry. The outside air passes through the middle of the two vortex rings. In the outside airflow movement process, some high-temperature and low-density air are brought out due to viscous action, and the flow field eventually evolves into a "mushroom cloud" shape.

\subsection{Flow Characteristics of the Laser Plasma under a Normal} Shock Wave. The experiment was conducted in a shock tube. The single-pulse laser energy is $82 \mathrm{~mJ}$, and the normal shock 


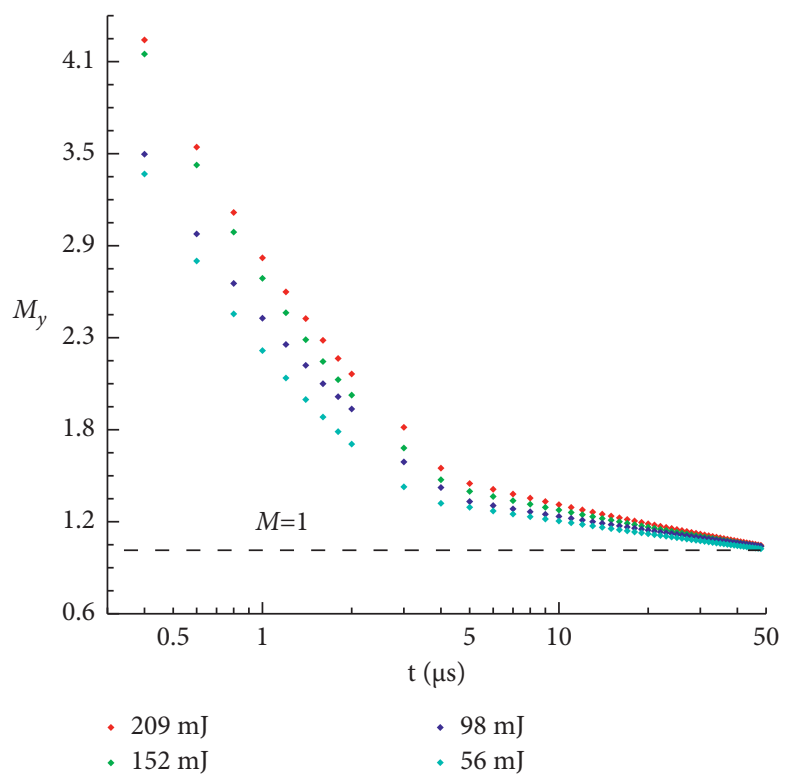

Figure 6: Shock wave propagation Mach numbers induced by a single-pulse laser plasma.
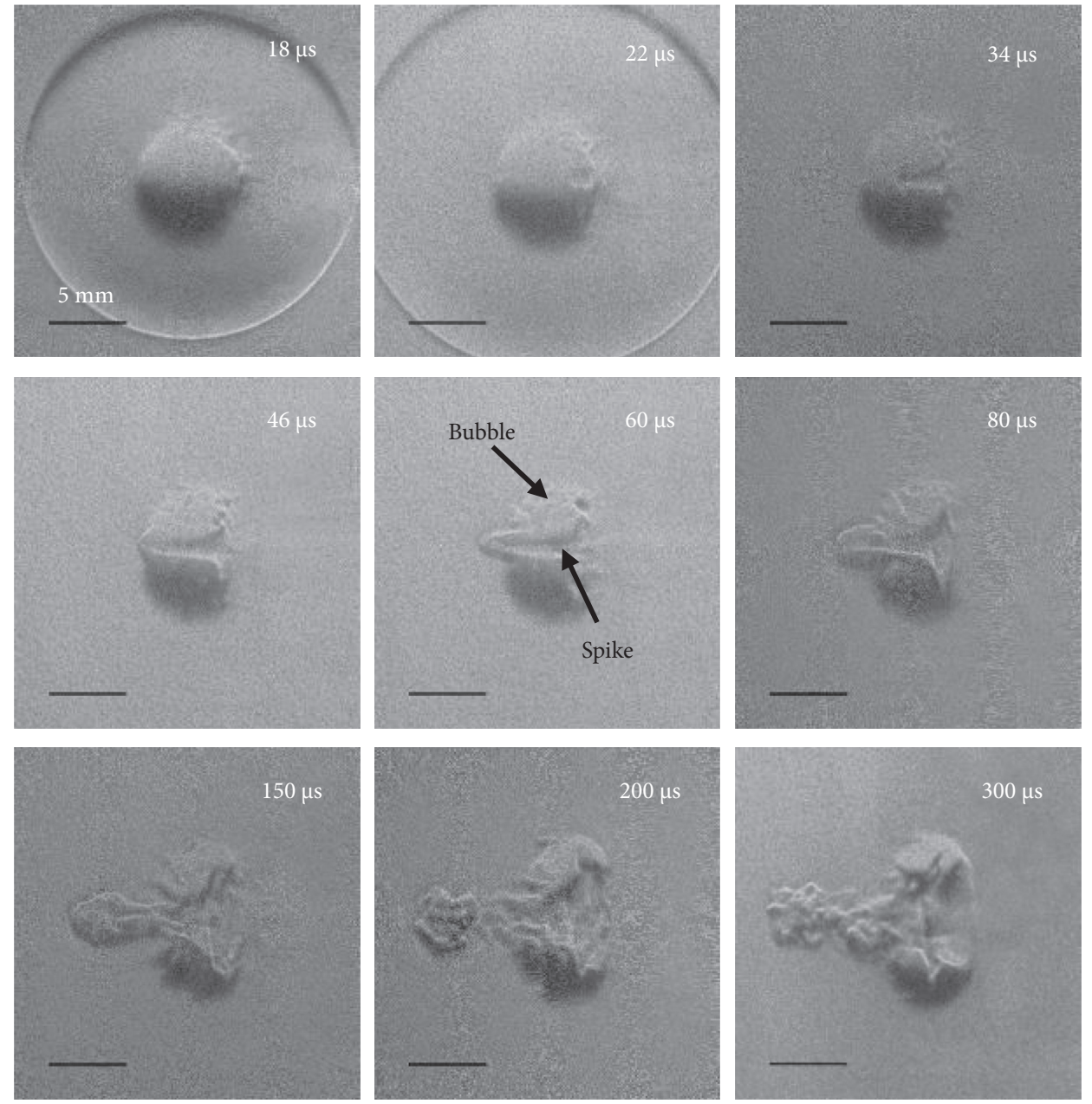

FiguRE 7: Schlieren photographs of the high-temperature and low-density region. 
Mach number is 1.45 . The experimental section is quiescent air with normal temperature and normal pressure. Two typical moments are selected to analyze the flow characteristics of the laser plasma under a normal shock, as shown in Figure 8. The left side is the test schlieren photograph, and the right side is the density gradient map at the corresponding time obtained by numerical calculation. In the present work, the numerical simulation method used in [22] was followed. However, for the sake of completeness, it is briefly described below. The grid was divided by ICEM software. The lengths of the high-pressure section and the low-pressure section were $0.2 \mathrm{~m}$ and $0.4 \mathrm{~m}$, respectively. The laser breakdown area was refined to 30 grids $/ \mathrm{mm}$ to improve the calculation efficiency, and the sparse grid was used in the rest of the area. The two-dimensional inviscid simulations were carried out with the commercial flow solver FLUENT. The shock tube model was used to generate the normal shock wave, and the UDF function of the Fluent software was used to realize the single pulse laser energy deposition in front of the normal shock wave. An asymmetric laser energy deposition model and a chemical reaction model were applied in the calculation to obtain more fitted simulation results for the experiment.

The normal shock wave propagates from right to left. At $t=40 \mu \mathrm{s}$, the positive shock wave has entered the spherical shock wave. It begins to interact with the high-temperature and low-density regions after the laser energy deposition occurs. After the normal shock wave, the air is compressed, the density increases, and the spherical shock wave propagation speed decreases. Therefore, the right side of the spherical shock wave is flatter than the left side. The normal shock wave entering the high-temperature and low-density region is faster, and it forms a hemispherical transmission shock wave (I), followed by the left side boundary (II) of the high-temperature low-density region. After the normal shock wave contacts the high-temperature and low-density region, the region's low-density air enters the normal shock wave to form a set of expansion waves (III). The middle curve (IV) is the boundary for the right side of the hightemperature and low-density region compressed by the normal shock wave. To the right of this boundary is the air that is not heated by the laser plasma. It has a relatively high density and a relatively low temperature, which is about to break through the boundary into the high-temperature and low-density regions. At $t=55 \mu \mathrm{s}$, the normal shock wave has passed through the high-temperature and low-density regions.

At the same time, those mentioned above "relatively high-density and relatively low-temperature" air, previously located to the right of the high-temperature and low-density region, now penetrate the region's central part. Under the action of the shear force, a vertically symmetrical vortex structure $(\mathrm{V})$ is formed, and it is foreseen that the size and vorticity of the vortex structure are anticipated to undergo further expansion. It should be noted that, in the schlieren photographs, it can always be seen that the normal shock wave is a straight line, which seems to contradict the above analysis and numerical calculation results. This is because the schlieren photograph shows the flow field density

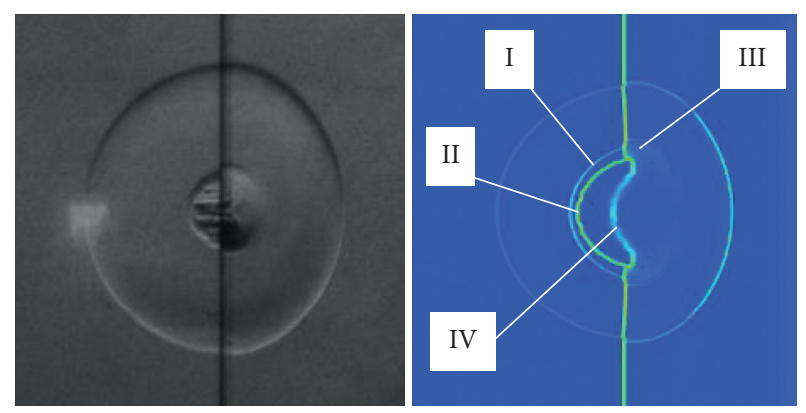

$t=40 \mu \mathrm{s}$

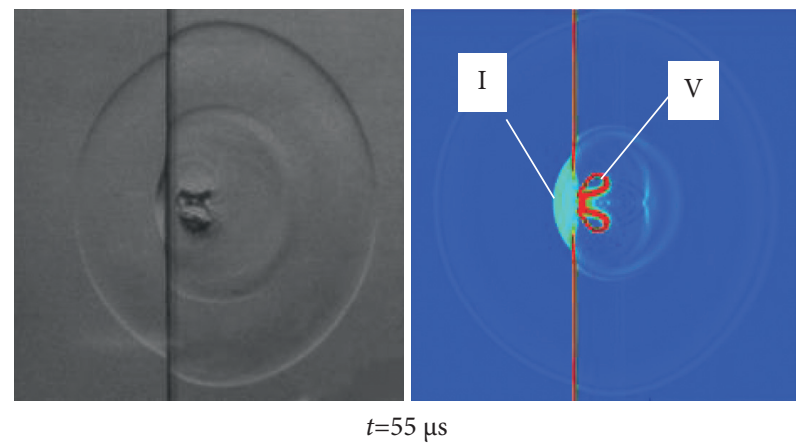

Figure 8: The schlieren results of the laser plasma under normal shock.

gradient along the optical path. The result of three-dimensional superposition includes the center that interacts with the high-temperature and low-density region and the parts to the left and the right that do not participate in the interaction. The parts that do not participate in the interaction appear as a completely straight line. However, the numerical calculations are two-dimensional results, which only demonstrate the part that participates in the interaction.

A high-speed color camera was used to build a color schlieren system for recording to observe the complete interaction between high-temperature and low-density region and normal shock waves. Figure 9 shows the results. When the injection time of the laser energy is taken as 0 , the time interval between two adjacent pictures is $24 \mu \mathrm{s}$, the exposure time of each picture is $0.28 \mu \mathrm{s}$, and the spatial resolution is $60.6 \mu \mathrm{m} /$ pixel. At $t=40 \mu \mathrm{s}$, the normal shock interacts with the high-temperature and low-density regions. The latter's flow structure is changed, and the details are as described above. At $t=64 \mu \mathrm{s}$, the normal shock has left the high-temperature and low-density region, which results in the formation of an asymmetric double-vortex ring structure. After $t=88 \mu \mathrm{s}$, the slip layer begins to evolve into a symmetrical secondary double-vortex ring structure.

Meanwhile, the size of the primary vortex ring and the secondary vortex ring is gradually increasing. Large-sized reflected wave structures also appear in the color schlieren photographs, which are formed by the reflection of the spherical shock caused by the laser plasma encountering the inner wall surface of the shock tube. It can also be seen from Figure 9 that the high-temperature and low-density region moves to the left because the quiescent air of the shock tube experimental section is swept by the normal shock wave. The 


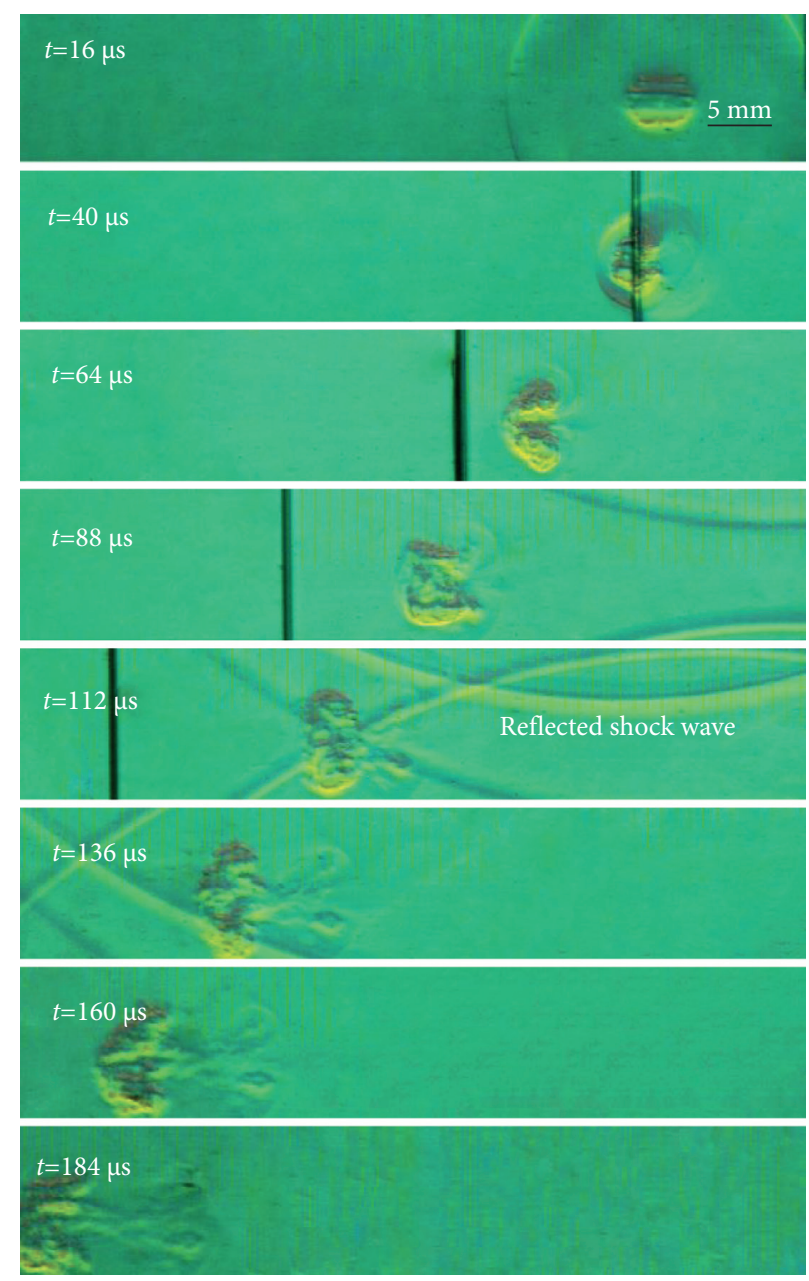

Figure 9: Color schlieren experiment results.

subsonic flow, accompanying the normal shock wave to the left, is generated. The high-temperature and low-density region move to the left as the flow moves.

Figure 10 shows two nanoparticle scattering images obtained by the PIV experiment with a time interval $\Delta t$ of $1 \mu \mathrm{s}$ and a spatial resolution of $45 \mu \mathrm{m} /$ pixel. The tracer particles in the high-temperature and low-density region and the vortex ring are less distributed because of the limited ability to follow the tracer particles in the high-speed airflow. Image processing is carried out by PIV VIEW software to obtain the velocity vector and vorticity map. The incident shock wave moves to the left, which is defined as the positive direction. From Figure 11(a), the velocity at the center of the high-temperature and low-density region is positive, the velocity at the upper and lower edges is negative, and the maximum velocity can exceed $200 \mathrm{~m} / \mathrm{s}$. It can be seen from Figure 11(b) that the vorticity is mainly concentrated downstream of the high-temperature and low-density region and exhibits a vertically symmetrical structure with a diameter of $4 \mathrm{~mm}$. The vorticity is caused by the shear force generated by the outside air penetrating the high-temperature and low-density area. This is consistent with the schlieren results. Figure 11(c) shows the vorticity distribution of the numerical simulation. The PIV results are roughly consistent with the vorticity distribution areas in the numerical simulation results. Moreover, in the numerical simulation results, except for the primary vortex ring (PVR), a secondary vortex ring (SVR) similar to the schlieren results can be seen.

Four kinds of single-pulse laser energy values of $48 \mathrm{~mJ}$, $82 \mathrm{~mJ}, 177 \mathrm{~mJ}$, and $256 \mathrm{~mJ}$ are selected to study the effect of laser energy on the interaction characteristics. The incident shock wave velocity is $590 \mathrm{~m} / \mathrm{s}$, and the Mach number is 1.74 . The first moment of action is $20 \mu \mathrm{s}$ after the laser energy deposition. After that, one image is taken every $24 \mu \mathrm{s}$, and the exposure time is $0.28 \mu \mathrm{s}$. The results of the color schlieren experiment are shown in Figure 12. It can be seen that, as the pulsed laser energy increases, the area of the high-temperature and low-density region increases at the same time, and the size of the corresponding vortex ring structure increases.

The experimental results of the wave drag reduction from the interaction between the nanosecond pulsed laser plasma and the shock wave lead to the following conjecture. Without the application of the laser plasma, when the shock wave occurs close to the supersonic body, the surface air pressure and density are high, which results in high drag. With the application of the laser plasma, a double-vortex structure is formed between the shock wave and the body. 

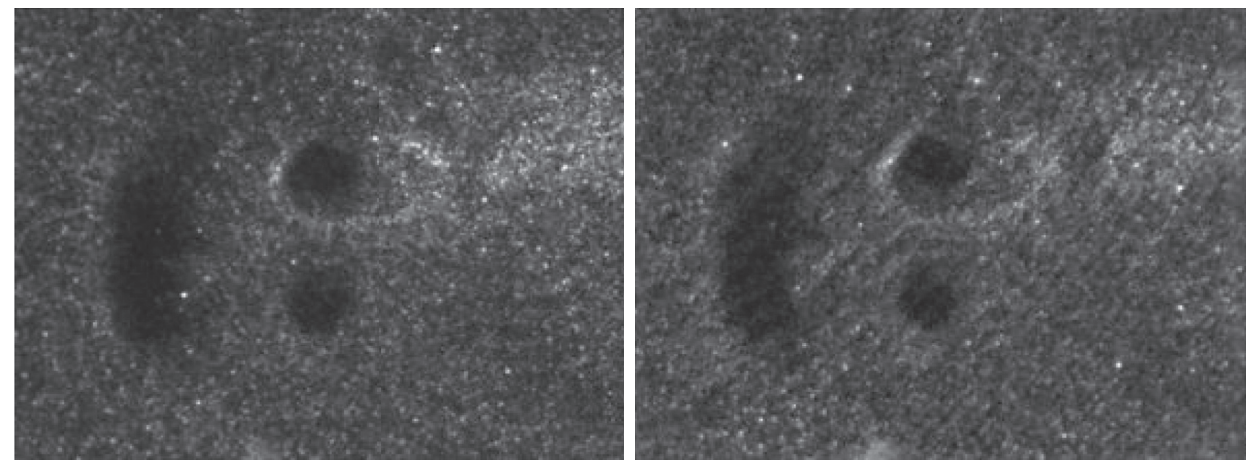

$t=80 \mathrm{~ms}, \Delta \mathrm{t}=1 \mu \mathrm{s}$

Figure 10: Nanoparticle scattering images.

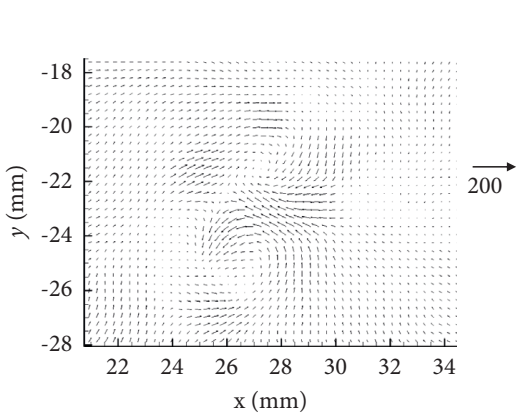

(a)

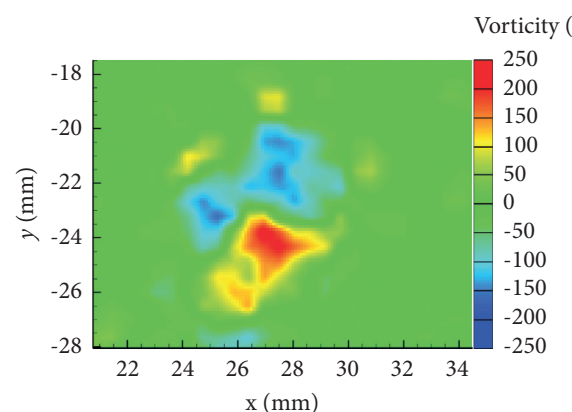

(b)

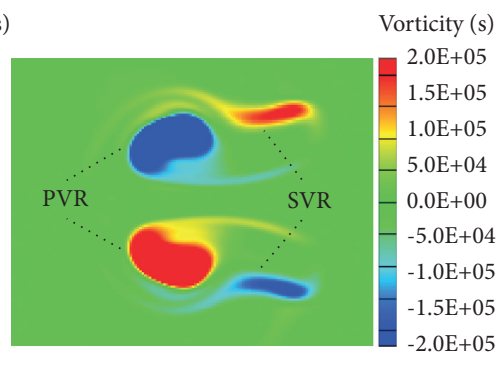

(c)

Figure 11: Velocity vector and vorticity distribution. (a) Velocity $(\mathrm{m} / \mathrm{s})$. (b) Vorticity $\left(\mathrm{s}^{-1}\right)$. (c) Vorticity $\left(\mathrm{s}^{-1}\right)$.

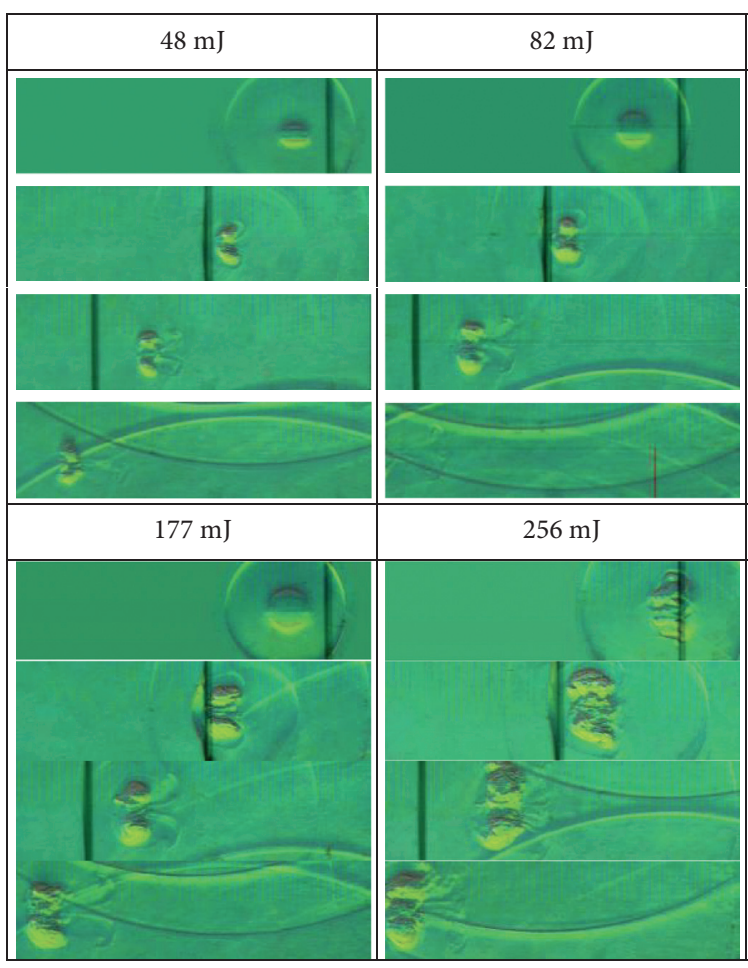

FIGURE 12: Effect of pulse laser energy. 
Meanwhile, the flow in the same direction as the flight direction between the double-vortex structures is reversed for the direction of the supersonic flow. Under the action of the entrainment and the backflow of the vortex structure, the high-pressure high-density gas on the surface of the body must be separated from the flight surface, causing a reduction in drag.

\section{Conclusion}

By adopting schlieren and PIV measurement methods, the characteristics of shock waves and high-temperature and low-density regions induced by the nanosecond pulsed laser plasma are studied experimentally in quiescent air and shock tube. Meanwhile, the flow mechanism and the evolution process under the impact of a normal shock are studied. The conclusions are as follows:

(1) The initial Mach number of the shock induced by the laser plasma increases with the laser energy; it can exceed 4.1. The shape gradually evolves from a droplet shape to a spherical shape. In addition, the propagation velocity decreases with time and approaches the sound velocity after $50 \mu \mathrm{s}$.

(2) The high-temperature and low-density region induced by the laser plasma is initially approximated as a spherical shape. The region destabilizes downstream of the incident direction of the laser due to the nonuniform laser energy deposition. It eventually forms a spike structure.

(3) Under the normal shock wave, the high-temperature and low-density region evolves into a double-vortex ring structure with upper and lower symmetry. The size of this structure increases with the laser energy.

\section{Data Availability}

No data were used to support this study.

\section{Conflicts of Interest}

The authors declare that they have no conflicts of interest.

\section{Acknowledgments}

This study was supported by Beijing Natural Science Foundation (1194028).

\section{References}

[1] L. N. Myrabo, "Solar-powered global air transportation// AIAA, DGLR," in Proceedings of the 13th International Electric Propulsion Conference, pp. 1-30, AIAA Paper, San Diego, CA, USA, April 1978.

[2] Z. Liang, C. Xiong, Z. Changsheng, and Y. Li, "Research on effects of lateral jet on drag and heat reduction characteristics of spike in supersonic flows," Journal of Propulsion Technology, vol. 39, no. 2, pp. 326-334, 2018.

[3] M. Gilinsky, I. M. Blankson, V. I. Sakhrov, and V. I. Shvets, "Shock waves mitigation at blunt bodies using needles and shells against a supersonic flow," AIAA Journal, vol. 3204, pp. 1-8, 2001.

[4] P. K. Tret'yakov, G. P. Grachev, A. I. Ivanchenko et al., "Stabilization of the optical discharge in a supersonic argon flow," Physics-Doklady, vol. 39, no. 6, pp. 415-416, 1994.

[5] P. K. Tret'yakov, A. F. Garanin, G. N. Grachev et al., "Control of supersonic flow around bodies by means of high-power recurrent optical breakdowns," Physics-Doklady, vol. 41, no. 11, pp. 566-567, 1996.

[6] R. Adelgren, G. Elliott, D. Knight et al., “T. energy deposition in supersonic flows//AIAA," in Proceedings of the 39th AIAA Aerospace Sciences meeting\&Exhibit, pp. 1-34, AIAA paper, Reno, NV, USA, January 2001.

[7] R. Adelgren, H. Yan, and G. Elliott, "Localized flow control by laser energy deposition applied to edney IV shock impingement and intersecting shocks//AIAA," in Proceedings of the 41st Aerospace Sciences Meeting and Exhibit, pp. 1-38, AIAA paper, Reno, NV, USA, January 2003.

[8] M. A. S. Minucci, P. G. P. Toro, J. B. Chanes et al., "Investigation of a laser-supported directed-energy "air spike" in hypersonic flow," Journal of Spacecraft and Rockets, vol. 40, no. 1, pp. 133-136, 2003.

[9] A. C. Oliveira, M. A. S. Minucci, P. G. P. Toro et al., "Schlieren visualization technique applied to the study of laser-induced breakdown in low density hypersonic flow//Kimiya Komurasaki," in Proceedings of the Fourth International Symposium on Beamed Energy Propulsion, T. Yabe, Ed., American Institute of Physics, Beamed Energy Propulsion, Nara, Japan, pp. 504-509, November 2005.

[10] A. C. Oliveira, M. A. S. Minucci, P. G. P. Toro, J. B. Chanes, L. N. Myrabo, and H. T. Nagamatsu, "Bow shock wave mitigation by laser-plasma energy addition in hypersonic flow," Journal of Spacecraft and Rockets, vol. 45, no. 5, pp. 921-927, 2008.

[11] R. M. Bracken, L. N. Myrabo, H. T. Nagamatsu et al., "Experimental/computational investigation of eclectric arc airspikes in hypersonic flow with drag measurements//AIAA," in Proceedings of the 37th AIAA/ASME/SAE/ASEE Joint Propulsion Conference, pp. 1-10, AIAA Paper, Salt Lake City, UT, Reston, VA, July 2001.

[12] D. Riggins, H. F. Nelson, and E. Johnson, "Blunt-body wave drag reduction using focused energy deposition," AIAA Journal, vol. 37, no. 4, pp. 460-467, 1999.

[13] S. Taguchi, N. Ohnishi, M. Furudate et al., "Numerical analysis of drag reduction for supersonic blunt body by pulse energy deposition//AIAA," in Proceedings of the 45th Aerospace Sciences Meeting and Exhibit, pp. 1-8, AIAA paper, Reno, Nevada, Reston, VA, January 2007.

[14] Y. Ogino, N. Ohnishi, S. Taguchi, and S. Keisuke, "Baroclinic vortex influence on wave drag reduction induced by pulse energy deposition," Physics of Fluids, vol. 21, no. 6, Article ID 0661021, 2009.

[15] X. Yu and H. Yan, "Parametric study of laser energy deposition in Mach 8 bow shock," International Journal of Flow Control, vol. 4, no. 1, pp. 19-28, 2012.

[16] X. J. Yu and H. Yan, "Effect of single- and repetitive-pulsed thermal actuator in a Mach 8 bow shock//AIAA," in Proceedings of the 51st AIAA Aerospace Science Meeting including the New Horizons Forum and Aerospace Exposition, pp. 1-6, AIAA Paper, Grapevine, TX, USA, January 2013.

[17] J. Fang, Y. Hong, and Q. Li, "Numerical analysis of interaction between single-pulse laser-induced plasma and bow shock in a supersonic flow," Plasma Science and Technology, vol. 14, no. 8, pp. 741-746, 2012. 
[18] D. Wang, Y. Hong, and Q. Li, "Investigation of stagnation pressure reduction of blunt body by laser energy deposition," Journal of Propulsion Tecnology, vol. 35, no. 2, pp. 172-177, 2014.

[19] A. Sasoh, Y. Sekiya, T. Sakai et al., "Supersonic drag reduction with repetitive laser pulses through a blunt body//AIAA," in Proceedings of the 40th Plasmadynamics and Lasers Conference, pp. 1-10, AIAA Paper, San Antonio, TX, USA, June 2009.

[20] O. Azarova, "Supersonic flow control using combined energy deposition," Aerospace, vol. 2, no. 1, pp. 118-134, 2015.

[21] J. H. J. Niederhaus, J. A. Greenough, J. G. Oakley, D. Ranjan, M. H. Anderson, and R. Bonazza, "A computational parameter study for the three-dimensional shock-bubble interaction," Journal of Fluid Mechanics, vol. 594, no. 594, pp. 85-124, 2008.

[22] D. Wang, J. Shi, and Z. Qing, "Numerical study of shock wave drag reduction mechanism by nanosecond-pulse laser energy deposition," Infrared and Laser Engineering, vol. 50, no. 3, pp. 155-164, 2021. 